Supporting information

\title{
Ring Cleavage of Aziridines by Difluoroamine: Mechanistic Insights from Ab initio and DFT study
}

\author{
Anbarasan Kalaiselvan, ${ }^{a}$ Salai Cheettu Ammal, ${ }^{\mathrm{b}}$ Ponnambalam Venuvanalingam, ${ }^{\mathrm{a}, *}$ \\ and Hiroshi Yamataka ${ }^{b} *$
}

${ }^{a}$ Department of Chemistry, Bharathidasan University, Tiruchirappalli-620 024, India and

${ }^{\mathrm{b}}$ Department of Chemistry, Rikkyo University, Nishi-Ikebukuro 3-34-1, Toshima-ku,

Tokyo 171-8501, Japan.

\section{CONTENTS}

I. Cartesian coordinates of reactants, complex, transition states, intermediates and products in the least energy pathway of deamination of cis-2,3-dimethylaziridine by $\mathrm{NHF}_{2}$ at HF/6-31G(d) [1-13], B3LYP/6-31G(d) [14-26], MP2/6-31G(d) [27-39] levels are given.

II. Cartesian coordinates and structures of the species in the high lying paths of deamination of cis-2,3-dimethylaziridine at B3LYP/6-31G(d) level are given.

"Author for correspondence: venuvanalingam@yahoo.com

I. HF/6-31G(d) optimized geometries

1. Difluoroamine

$\begin{array}{rrrr}7 & 0.000001 & 0.544266 & -0.162516 \\ 1 & 0.000011 & 1.076588 & 0.688389 \\ 9 & 1.063936 & -0.271470 & 0.024956 \\ 9 & -1.063938 & -0.271468 & 0.024957\end{array}$

2. cis-2,3-dimethylaziridine

$\begin{array}{lrrr}6 & 0.737662 & -0.370890 & -0.412000 \\ 6 & -0.737665 & -0.370895 & -0.411999 \\ 7 & 0.000009 & -1.224156 & 0.506176 \\ 1 & 1.216564 & -0.932440 & -1.198061 \\ 1 & -1.216549 & -0.932424 & -1.198088\end{array}$




$\begin{array}{rrrr}6 & 1.584145 & 0.776245 & 0.089778 \\ 6 & -1.584156 & 0.776231 & 0.089785 \\ 1 & 2.536449 & 0.404577 & 0.455901 \\ 1 & 1.789341 & 1.479992 & -0.711455 \\ 1 & 1.111155 & 1.320369 & 0.898909 \\ 1 & -1.111175 & 1.320347 & 0.898926 \\ 1 & -1.789346 & 1.479986 & -0.711441 \\ 1 & -2.536464 & 0.404557 & 0.455895 \\ 1 & 0.000051 & -0.840012 & 1.432801\end{array}$

3. Complex

$\begin{array}{rrrr}6 & 1.246056 & -0.771767 & -0.178058 \\ 6 & 0.892579 & 0.650163 & -0.328614 \\ 7 & 0.521134 & -0.077426 & 0.882991 \\ 1 & 0.620112 & -1.472799 & -0.703257 \\ 1 & 0.037300 & 0.862532 & -0.947265 \\ 6 & 2.650115 & -1.278881 & 0.051940 \\ 6 & 1.887189 & 1.785439 & -0.273678 \\ 1 & 2.623847 & -2.213140 & 0.603965 \\ 1 & 3.143732 & -1.467680 & -0.896175 \\ 1 & 3.259588 & -0.580243 & 0.612822 \\ 1 & 2.723808 & 1.576262 & 0.382496 \\ 1 & 2.281297 & 1.990739 & -1.263985 \\ 1 & 1.402361 & 2.688200 & 0.084026 \\ 1 & 1.126724 & 0.154062 & 1.648235 \\ 7 & -2.403907 & -0.379760 & 0.464204 \\ 9 & -2.097785 & -0.954960 & -0.734460 \\ 9 & -2.636603 & 0.917999 & 0.127843 \\ 1 & -1.485501 & -0.314694 & 0.878785\end{array}$

4. $\underline{\mathrm{TS} 1}$

$\begin{array}{lccc}6 & -1.195867 & -0.738178 & 0.323233 \\ 6 & -1.065897 & 0.726680 & 0.453866 \\ 7 & -0.236525 & -0.010482 & -0.496508 \\ 1 & -0.701522 & -1.315127 & 1.084682 \\ 1 & -0.487795 & 1.063151 & 1.297027 \\ 6 & -2.357321 & -1.446125 & -0.330655 \\ 6 & -2.072815 & 1.733668 & -0.046393 \\ 1 & -2.026727 & -2.388589 & -0.752837 \\ 1 & -3.120745 & -1.660016 & 0.409356 \\ 1 & -2.810854 & -0.862497 & -1.122269 \\ 1 & -2.608534 & 1.388824 & -0.922064 \\ 1 & -2.798970 & 1.943560 & 0.731372 \\ 1 & -1.576206 & 2.664047 & -0.299162 \\ 1 & -0.497203 & 0.084158 & -1.460158 \\ 7 & 1.597427 & -0.314785 & -0.322662 \\ 9 & 1.595527 & -0.382827 & 1.002335 \\ 9 & 3.438236 & 0.281660 & -0.457716\end{array}$


5. $\underline{\text { Int1 }}$

$\begin{array}{lrrr}6 & -0.406138 & 0.744404 & -0.399908 \\ 6 & -0.406150 & -0.744430 & -0.399894 \\ 7 & 0.335763 & -0.000012 & 0.613459 \\ 1 & 0.270620 & 1.192192 & -1.100963 \\ 1 & 0.270591 & -1.192244 & -1.100949 \\ 6 & -1.569455 & 1.608853 & 0.017778 \\ 6 & -1.569533 & -1.608803 & 0.017777 \\ 1 & -1.208717 & 2.556831 & 0.401360 \\ 1 & -2.201741 & 1.812497 & -0.839808 \\ 1 & -2.184278 & 1.147306 & 0.781747 \\ 1 & -2.183633 & -1.147708 & 0.782604 \\ 1 & -2.202501 & -1.811409 & -0.839553 \\ 1 & -1.208920 & -2.557262 & 0.400272 \\ 1 & -0.068922 & -0.000003 & 1.530383 \\ 7 & 1.774020 & -0.000010 & 0.791027 \\ 9 & 2.184075 & -0.000022 & -0.584556\end{array}$

6. $\underline{\mathrm{TS} 2}$

$\begin{array}{rrrr}6 & 0.654221 & -0.687861 & -0.477640 \\ 6 & 0.255008 & 0.732259 & -0.379661 \\ 7 & -0.310527 & -0.259898 & 0.539907 \\ 1 & 0.142926 & -1.250225 & -1.237128 \\ 1 & -0.485219 & 1.050138 & -1.083421 \\ 6 & 1.988293 & -1.246983 & -0.051225 \\ 6 & 1.115581 & 1.836453 & 0.182544 \\ 1 & 1.880876 & -2.287042 & 0.235681 \\ 1 & 2.690473 & -1.196376 & -0.876328 \\ 1 & 2.419286 & -0.708696 & 0.784637 \\ 1 & 1.804416 & 1.491516 & 0.944149 \\ 1 & 1.697642 & 2.282872 & -0.616453 \\ 1 & 0.489774 & 2.609011 & 0.614438 \\ 1 & 0.051836 & -0.208803 & 1.472978 \\ 7 & -1.700719 & -0.709301 & 0.579806 \\ 9 & -2.299101 & 0.133199 & -0.413405\end{array}$

7. $\underline{\text { Int2 }}$

$\begin{array}{lrrc}6 & -0.859045 & -0.664581 & 0.469706 \\ 6 & -0.268508 & 0.687765 & 0.526514 \\ 7 & 0.336202 & -0.351849 & -0.314791 \\ 1 & -0.593051 & -1.313843 & 1.281805 \\ 1 & 0.369759 & 0.876132 & 1.367431 \\ 6 & -2.136045 & -1.025507 & -0.246840 \\ 6 & -0.852265 & 1.913597 & -0.128491\end{array}$




$\begin{array}{rrrr}1 & -2.096796 & -2.056288 & -0.580108 \\ 1 & -2.972486 & -0.923893 & 0.435711 \\ 1 & -2.330092 & -0.397477 & -1.107148 \\ 1 & -1.416568 & 1.686113 & -1.024663 \\ 1 & -1.517400 & 2.417349 & 0.564397 \\ 1 & -0.055736 & 2.599060 & -0.393282 \\ 1 & 0.198060 & -0.233746 & -1.305634 \\ 7 & 1.541509 & -0.972818 & 0.088942 \\ 9 & 2.440612 & 0.127957 & -0.153655\end{array}$

8. $\underline{\text { TS3 }}$

$\begin{array}{rrrr}6 & 0.731854 & 0.750094 & 0.571584 \\ 6 & 0.731937 & -0.750095 & 0.571547 \\ 7 & -0.437383 & -0.000052 & 0.184046 \\ 1 & 0.628790 & 1.194041 & 1.547557 \\ 1 & 0.628939 & -1.194098 & 1.547504 \\ 6 & 1.426189 & 1.619130 & -0.446990 \\ 6 & 1.426416 & -1.618979 & -0.447063 \\ 1 & 0.910760 & 2.568816 & -0.536252 \\ 1 & 2.446813 & 1.814974 & -0.135702 \\ 1 & 1.450961 & 1.157702 & -1.425847 \\ 1 & 1.450771 & -1.157666 & -1.425984 \\ 1 & 2.447193 & -1.814390 & -0.135995 \\ 1 & 0.911336 & -2.568874 & -0.536087 \\ 1 & -1.114231 & -0.000024 & -0.783398 \\ 7 & -1.660273 & -0.000118 & 0.865269 \\ 9 & -2.330680 & -0.000022 & -0.772829\end{array}$

9. Int3

$\begin{array}{lrrr}6 & 0.768320 & -0.153036 & 0.596567 \\ 6 & -0.768335 & -0.153000 & 0.596566 \\ 7 & 0.000019 & 0.940721 & 0.087885 \\ 1 & 1.215574 & 0.032069 & 1.560537 \\ 1 & -1.215582 & 0.032123 & 1.560535 \\ 6 & 1.615497 & -0.977077 & -0.342294 \\ 6 & -1.615537 & -0.977015 & -0.342297 \\ 1 & 2.576112 & -0.495392 & -0.488494 \\ 1 & 1.791650 & -1.964730 & 0.071673 \\ 1 & 1.150739 & -1.088458 & -1.313398 \\ 1 & -1.150884 & -1.088210 & -1.313474 \\ 1 & -1.791527 & -1.964744 & 0.071560 \\ 1 & -2.576224 & -0.495421 & -0.488318 \\ 7 & 0.000048 & 2.001212 & -0.475295\end{array}$

10. $\underline{\mathrm{TS} 4}$
6
$\begin{array}{lll}0.670686 & -0.297510 & 0.576809\end{array}$
6
$\begin{array}{lll}-0.797136 & -0.363953 & 0.721286\end{array}$ 


$\begin{array}{rrrr}7 & 0.366354 & 1.023461 & 0.130215 \\ 1 & 1.182376 & -0.219863 & 1.524579 \\ 1 & -1.184211 & -0.194390 & 1.706740 \\ 6 & 1.443519 & -1.132901 & -0.425272 \\ 6 & -1.733431 & -0.638328 & -0.413313 \\ 1 & 2.461480 & -0.775560 & -0.530580 \\ 1 & 1.461443 & -2.153302 & -0.058455 \\ 1 & 0.967669 & -1.125553 & -1.396692 \\ 1 & -1.912902 & 0.249614 & -1.033823 \\ 1 & -1.396674 & -1.429640 & -1.080615 \\ 1 & -2.699613 & -0.944094 & -0.026509 \\ 7 & 0.150590 & 2.003531 & -0.396172\end{array}$

11. cis-2-butene

$\begin{array}{lrrr}6 & 0.661059 & 0.659707 & 0.000017 \\ 6 & -0.661060 & 0.659708 & -0.000045 \\ 6 & -1.594169 & -0.520179 & -0.000002 \\ 6 & 1.594169 & -0.520179 & -0.000002 \\ 1 & 1.153573 & 1.619736 & 0.000046 \\ 1 & -1.153573 & 1.619737 & 0.000025 \\ 1 & -1.075901 & -1.470097 & -0.003516 \\ 1 & -2.243428 & -0.491494 & -0.871529 \\ 1 & -2.238736 & -0.495319 & 0.875148 \\ 1 & 1.075899 & -1.470100 & 0.001607 \\ 1 & 2.240058 & -0.494273 & -0.874138 \\ 1 & 2.242110 & -0.492534 & 0.872548\end{array}$

12. Nitrogen

$\begin{array}{rrrr}7 & 0.000000 & 0.000000 & 0.539188 \\ 7 & 0.000000 & 0.000000 & -0.539188\end{array}$

13. $\underline{\mathrm{HF}}$
9
$\begin{array}{lll}0.000000 & 0.000000 & 0.091095\end{array}$
$\begin{array}{llll}0.000000 & 0.000000 & -0.819855\end{array}$

\section{B3LYP/6-31G(d) optimized geometries}

14. Difluoroamine

$\begin{array}{rrrr}7 & 0.000391 & 0.567339 & -0.166728 \\ 9 & -1.103911 & -0.279325 & 0.023772 \\ 9 & 1.103616 & -0.279546 & 0.023831 \\ 1 & -0.000090 & 1.058469 & 0.738669\end{array}$


15. cis-2,3-dimethylaziridine

$\begin{array}{lrrr}7 & 0.000000 & -1.247692 & 0.508385 \\ 6 & 0.745521 & -0.366494 & -0.417161 \\ 6 & -0.745522 & -0.366494 & -0.417161 \\ 6 & -1.590851 & 0.779735 & 0.092384 \\ 6 & 1.590852 & 0.779734 & 0.092384 \\ 1 & 0.000000 & -0.826100 & 1.440787 \\ 1 & 1.227014 & -0.930157 & -1.217554 \\ 1 & -1.227016 & -0.930156 & -1.217553 \\ 1 & -1.090323 & 1.350709 & 0.881460 \\ 1 & -1.837165 & 1.475504 & -0.719396 \\ 1 & -2.535634 & 0.404473 & 0.504415 \\ 1 & 2.535631 & 0.404471 & 0.504425 \\ 1 & 1.090322 & 1.350716 & 0.881453 \\ 1 & 1.837175 & 1.475497 & -0.719399\end{array}$

16. Complex

$\begin{array}{rrrr}6 & 1.651326 & -0.590079 & -0.710896 \\ 6 & 1.428301 & 0.864532 & -0.470460 \\ 7 & 0.356046 & -0.112630 & -0.174668 \\ 1 & 1.565700 & -0.922405 & -1.745469 \\ 1 & 1.199591 & 1.465402 & -1.350611 \\ 6 & 2.563119 & -1.458157 & 0.126171 \\ 6 & 2.087281 & 1.651292 & 0.639721 \\ 1 & 2.196617 & -2.491219 & 0.143867 \\ 1 & 3.575877 & -1.471367 & -0.293946 \\ 1 & 2.634059 & -1.112633 & 1.162421 \\ 1 & 2.312177 & 1.032936 & 1.514553 \\ 1 & 3.027413 & 2.095481 & 0.291598 \\ 1 & 1.434328 & 2.468377 & 0.968017 \\ 1 & 0.304413 & -0.285370 & 0.831199 \\ 7 & -2.493657 & -0.488013 & -0.371709 \\ 9 & -3.032500 & 0.814323 & -0.419159 \\ 9 & -2.319564 & -0.714561 & 1.019021 \\ 1 & -1.498490 & -0.278087 & -0.602973\end{array}$

17. $\underline{\mathrm{TS} 1}$

$\begin{array}{lrrr}6 & -1.211083 & -0.747801 & 0.294064 \\ 6 & -1.058162 & 0.736735 & 0.435305 \\ 7 & -0.262413 & -0.011955 & -0.551406 \\ 1 & -0.681326 & -1.334538 & 1.042646 \\ 1 & -0.433885 & 1.051460 & 1.270570 \\ 6 & -2.405058 & -1.435922 & -0.323988 \\ 6 & -2.073420 & 1.758524 & -0.018674 \\ 1 & -2.097953 & -2.372029 & -0.803138 \\ 1 & -3.137845 & -1.683030 & 0.452263 \\ 1 & -2.905771 & -0.816947 & -1.074839\end{array}$




$\begin{array}{rrrr}1 & -2.675444 & 1.408377 & -0.862799 \\ 1 & -2.755541 & 1.998226 & 0.804745 \\ 1 & -1.574219 & 2.686680 & -0.318327 \\ 1 & -0.529347 & 0.095638 & -1.529331 \\ 7 & 1.622758 & -0.342937 & -0.328392 \\ 9 & 1.569795 & -0.403835 & 1.064265 \\ 9 & 3.500851 & 0.292224 & -0.470289 \\ 1 & 2.119443 & 0.585680 & -0.489226\end{array}$

18. Int1

$\begin{array}{lrrr}6 & -0.420747 & 0.755095 & -0.407999 \\ 6 & -0.420873 & -0.755166 & -0.408016 \\ 7 & 0.362286 & -0.000116 & 0.628609 \\ 1 & 0.270010 & 1.198689 & -1.117703 \\ 1 & 0.269779 & -1.198918 & -1.117717 \\ 6 & -1.583221 & 1.615032 & 0.017985 \\ 6 & -1.583628 & -1.614748 & 0.017925 \\ 1 & -1.225816 & 2.574758 & 0.407218 \\ 1 & -2.235289 & 1.824498 & -0.838678 \\ 1 & -2.198250 & 1.142819 & 0.791605 \\ 1 & -2.196739 & -1.143610 & 0.793730 \\ 1 & -2.237459 & -1.821439 & -0.838075 \\ 1 & -1.226684 & -2.575767 & 0.404347 \\ 1 & -0.053477 & -0.000154 & 1.559566 \\ 7 & 1.781351 & -0.000147 & 0.808420 \\ 9 & 2.208808 & -0.000035 & -0.602539\end{array}$

19. $\mathrm{TS} 2$

$\begin{array}{lrrr}6 & 0.758899 & -0.678226 & -0.512876 \\ 6 & 0.214052 & 0.705219 & -0.379603 \\ 7 & -0.326498 & -0.332171 & 0.538856 \\ 1 & 0.295176 & -1.283698 & -1.286435 \\ 1 & -0.558852 & 0.964905 & -1.093477 \\ 6 & 2.120654 & -1.111559 & -0.036926 \\ 6 & 0.967528 & 1.878313 & 0.199029 \\ 1 & 2.132352 & -2.187859 & 0.166073 \\ 1 & 2.883174 & -0.905549 & -0.798205 \\ 1 & 2.426827 & -0.591546 & 0.877824 \\ 1 & 1.699496 & 1.585188 & 0.958037 \\ 1 & 1.507055 & 2.397585 & -0.601470 \\ 1 & 0.269327 & 2.590793 & 0.650442 \\ 1 & 0.065004 & -0.284846 & 1.482667 \\ 7 & -1.677686 & -0.807618 & 0.551861 \\ 9 & -2.339675 & 0.103451 & -0.400913\end{array}$


20. Int2

$\begin{array}{lrrc}6 & -0.847317 & -0.678858 & 0.473742 \\ 6 & -0.329763 & 0.719412 & 0.550373 \\ 7 & 0.377203 & -0.342387 & -0.298496 \\ 1 & -0.563313 & -1.316523 & 1.305761 \\ 1 & 0.300439 & 0.933588 & 1.406791 \\ 6 & -2.093001 & -1.101924 & -0.266817 \\ 6 & -0.958674 & 1.902111 & -0.139370 \\ 1 & -1.993275 & -2.133251 & -0.621215 \\ 1 & -2.950324 & -1.060956 & 0.414071 \\ 1 & -2.319321 & -0.463251 & -1.125295 \\ 1 & -1.448673 & 1.631794 & -1.080511 \\ 1 & -1.717356 & 2.360694 & 0.505682 \\ 1 & -0.197706 & 2.658735 & -0.357330 \\ 1 & 0.215656 & -0.181579 & -1.310130 \\ 7 & 1.562977 & -0.942447 & 0.119184 \\ 9 & 2.496127 & 0.168905 & -0.176690\end{array}$

21. $\underline{\mathrm{TS} 3}$

$\begin{array}{rrrr}6 & 0.751700 & 0.761902 & 0.574607 \\ 6 & 0.751742 & -0.761918 & 0.574564 \\ 7 & -0.446946 & -0.000050 & 0.194352 \\ 1 & 0.656896 & 1.208682 & 1.563978 \\ 1 & 0.656958 & -1.208756 & 1.563912 \\ 6 & 1.448411 & 1.621771 & -0.451838 \\ 6 & 1.448580 & -1.621653 & -0.451909 \\ 1 & 0.928118 & 2.580029 & -0.558802 \\ 1 & 2.479440 & 1.830066 & -0.142433 \\ 1 & 1.478471 & 1.143272 & -1.435079 \\ 1 & 1.477155 & -1.143774 & -1.435498 \\ 1 & 2.480143 & -1.828498 & -0.143300 \\ 1 & 0.929340 & -2.580599 & -0.557773 \\ 1 & -1.196575 & 0.000014 & -0.816656 \\ 7 & -1.680678 & -0.000102 & 0.853296 \\ 9 & -2.377686 & 0.000001 & -0.760493\end{array}$

22. Int3

$\begin{array}{lrrr}6 & 0.778670 & -0.178267 & 0.607349 \\ 6 & -0.778845 & -0.177761 & 0.607309 \\ 7 & 0.000321 & 0.971194 & 0.099560 \\ 1 & 1.225555 & -0.006166 & 1.586928 \\ 1 & -1.225635 & -0.005335 & 1.586873 \\ 6 & 1.621911 & -0.980514 & -0.353605 \\ 6 & -1.622544 & -0.979547 & -0.353618 \\ 1 & 2.594526 & -0.496279 & -0.494279 \\ 1 & 1.798740 & -1.992447 & 0.030775 \\ 1 & 1.149128 & -1.064447 & -1.336478\end{array}$




$\begin{array}{lrrr}1 & -1.150208 & -1.063036 & -1.336747 \\ 1 & -1.799229 & -1.991656 & 0.030373 \\ 1 & -2.595206 & -0.495227 & -0.493645 \\ 7 & 0.000705 & 2.030396 & -0.473617\end{array}$

23. TS4

$\begin{array}{lrrr}6 & 0.561661 & -0.449689 & 0.569880 \\ 6 & -0.913458 & -0.157636 & 0.719360 \\ 7 & 0.596537 & 0.892285 & 0.140905 \\ 1 & 1.068252 & -0.523162 & 1.538372 \\ 1 & -1.240146 & 0.149017 & 1.705405 \\ 6 & 1.087905 & -1.490026 & -0.412177 \\ 6 & -1.870558 & -0.152956 & -0.430434 \\ 1 & 2.175473 & -1.427071 & -0.517772 \\ 1 & 0.820971 & -2.480248 & -0.027006 \\ 1 & 0.630386 & -1.369664 & -1.397840 \\ 1 & -1.826455 & 0.775053 & -1.034853 \\ 1 & -1.718970 & -0.991068 & -1.126060 \\ 1 & -2.898733 & -0.231931 & -0.059984 \\ 7 & 0.802881 & 1.907846 & -0.392339\end{array}$

24. cis-2-butene

$\begin{array}{lrrc}6 & 0.669212 & 0.664153 & 0.000056 \\ 6 & -0.669226 & 0.664165 & -0.000099 \\ 6 & -1.592824 & -0.521710 & -0.000010 \\ 6 & 1.592829 & -0.521702 & -0.000005 \\ 1 & 1.169627 & 1.633345 & 0.000119 \\ 1 & -1.169624 & 1.633363 & 0.000009 \\ 1 & -1.060135 & -1.476696 & -0.006108 \\ 1 & -2.254542 & -0.502394 & -0.876815 \\ 1 & -2.246220 & -0.509007 & 0.883206 \\ 1 & 1.060170 & -1.476721 & 0.002874 \\ 1 & 2.248500 & -0.507202 & -0.881488 \\ 1 & 2.252286 & -0.504123 & 0.878559\end{array}$

25. Nitrogen

$\begin{array}{rrrr}7 & 0.000000 & 0.000000 & 0.552800 \\ 7 & 0.000000 & 0.000000 & -0.552800\end{array}$

26. $\underline{\mathrm{HF}}$

$\begin{array}{rrrr}9 & 0.000000 & 0.000000 & 0.093380 \\ 1 & 0.000000 & 0.000000 & -0.840421\end{array}$




\section{MP2/6-31G(d) optimized geometries}

27. Difluoroamine

$\begin{array}{rrrr}7 & 0.000024 & 0.571934 & -0.165950 \\ 9 & -1.102650 & -0.280963 & 0.023295 \\ 9 & 1.102639 & -0.280968 & 0.023304 \\ 1 & -0.000067 & 1.053839 & 0.742259\end{array}$

28. cis-2,3-dimethylaziridine

$\begin{array}{lrrr}7 & 0.000000 & -1.249376 & 0.509235 \\ 6 & 0.742301 & -0.365941 & -0.418551 \\ 6 & -0.742301 & -0.365941 & -0.418551 \\ 6 & -1.576599 & 0.780270 & 0.092878 \\ 6 & 1.576599 & 0.780270 & 0.092878 \\ 1 & 0.000000 & -0.814690 & 1.437290 \\ 1 & 1.222846 & -0.930259 & -1.216798 \\ 1 & -1.222846 & -0.930259 & -1.216798 \\ 1 & -1.076635 & 1.323291 & 0.898487 \\ 1 & -1.794086 & 1.491493 & -0.710635 \\ 1 & -2.530052 & 0.409662 & 0.482013 \\ 1 & 2.530052 & 0.409662 & 0.482013 \\ 1 & 1.076635 & 1.323291 & 0.898487 \\ 1 & 1.794086 & 1.491493 & -0.710635\end{array}$

29. Complex

$\begin{array}{lrrr}6 & 1.682405 & -0.581750 & -0.703974 \\ 6 & 1.385153 & 0.854091 & -0.468927 \\ 7 & 0.357845 & -0.173831 & -0.180757 \\ 1 & 1.622525 & -0.919064 & -1.737527 \\ 1 & 1.133661 & 1.440540 & -1.351183 \\ 6 & 2.624944 & -1.392831 & 0.145933 \\ 6 & 1.991131 & 1.664845 & 0.646342 \\ 1 & 2.314830 & -2.441971 & 0.157690 \\ 1 & 3.641802 & -1.344923 & -0.256006 \\ 1 & 2.651925 & -1.039632 & 1.179229 \\ 1 & 2.217498 & 1.052519 & 1.522164 \\ 1 & 2.919628 & 2.140045 & 0.314816 \\ 1 & 1.299345 & 2.453199 & 0.957501 \\ 1 & 0.321770 & -0.344211 & 0.828531 \\ 7 & -2.510406 & -0.482334 & -0.403696 \\ 9 & -2.947623 & 0.861376 & -0.374537 \\ 9 & -2.346625 & -0.787843 & 0.973509 \\ 1 & -1.508630 & -0.331276 & -0.631047\end{array}$


30. $\underline{T S 1}$

$\begin{array}{lrrc}6 & -1.170865 & -0.746874 & 0.327515 \\ 6 & -1.032061 & 0.738223 & 0.419262 \\ 7 & -0.226164 & -0.033964 & -0.541575 \\ 1 & -0.636674 & -1.302946 & 1.094950 \\ 1 & -0.409746 & 1.082922 & 1.242589 \\ 6 & -2.352395 & -1.453760 & -0.279349 \\ 6 & -2.048621 & 1.728025 & -0.080492 \\ 1 & -2.038154 & -2.406850 & -0.713513 \\ 1 & -3.097183 & -1.662335 & 0.494131 \\ 1 & -2.832603 & -0.861431 & -1.061437 \\ 1 & -2.622198 & 1.342986 & -0.926276 \\ 1 & -2.749471 & 1.975569 & 0.722071 \\ 1 & -1.552678 & 2.651291 & -0.392831 \\ 1 & -0.493150 & 0.035299 & -1.527025 \\ 7 & 1.569507 & -0.312987 & -0.344606 \\ 9 & 1.523892 & -0.264077 & 1.071540 \\ 9 & 3.431836 & 0.195187 & -0.459022 \\ 1 & 2.050563 & 0.600481 & -0.563674\end{array}$

31. Int1

$\begin{array}{lrrr}6 & -0.409178 & 0.753011 & -0.401032 \\ 6 & -0.409214 & -0.753032 & -0.401037 \\ 7 & 0.349982 & -0.000032 & 0.629290 \\ 1 & 0.288030 & 1.196262 & -1.104865 \\ 1 & 0.287962 & -1.196321 & -1.104877 \\ 6 & -1.576965 & 1.603530 & 0.013749 \\ 6 & -1.577083 & -1.603448 & 0.013729 \\ 1 & -1.224403 & 2.561452 & 0.406053 \\ 1 & -2.218543 & 1.805328 & -0.849367 \\ 1 & -2.190242 & 1.123958 & 0.780932 \\ 1 & -2.189784 & -1.124214 & 0.781587 \\ 1 & -2.219195 & -1.804409 & -0.849187 \\ 1 & -1.224650 & -2.561759 & 0.405190 \\ 1 & -0.073361 & -0.000037 & 1.560798 \\ 7 & 1.778385 & -0.000045 & 0.809719 \\ 9 & 2.188919 & -0.000010 & -0.605753\end{array}$

32. $\underline{\mathrm{TS} 2}$

$\begin{array}{lrrr}6 & 0.702410 & -0.687711 & -0.492778 \\ 6 & 0.230772 & 0.722922 & -0.376163 \\ 7 & -0.313524 & -0.303050 & 0.545542 \\ 1 & 0.198905 & -1.274499 & -1.257089 \\ 1 & -0.541428 & 1.008595 & -1.080051 \\ 6 & 2.055884 & -1.171901 & -0.054576 \\ 6 & 1.044694 & 1.852503 & 0.192793\end{array}$




$\begin{array}{lrrr}1 & 2.011232 & -2.232831 & 0.206031 \\ 1 & 2.782121 & -1.049415 & -0.863730 \\ 1 & 2.428942 & -0.621779 & 0.813145 \\ 1 & 1.763193 & 1.517862 & 0.944467 \\ 1 & 1.601850 & 2.341641 & -0.611663 \\ 1 & 0.384843 & 2.594574 & 0.649965 \\ 1 & 0.075300 & -0.246936 & 1.491890 \\ 7 & -1.689935 & -0.766184 & 0.571726 \\ 9 & -2.320368 & 0.128061 & -0.414388\end{array}$

33. Int2

$\begin{array}{lrrr}6 & -0.864264 & -0.670118 & 0.470803 \\ 6 & -0.269676 & 0.694757 & 0.530420 \\ 7 & 0.346173 & -0.367766 & -0.329906 \\ 1 & -0.579027 & -1.328033 & 1.286197 \\ 1 & 0.396187 & 0.872836 & 1.368507 \\ 6 & -2.144380 & -1.015419 & -0.238580 \\ 6 & -0.859863 & 1.909192 & -0.128227 \\ 1 & -2.112655 & -2.050107 & -0.589948 \\ 1 & -2.981849 & -0.916767 & 0.457821 \\ 1 & -2.340674 & -0.365560 & -1.093530 \\ 1 & -1.431921 & 1.664121 & -1.025997 \\ 1 & -1.531798 & 2.421512 & 0.566519 \\ 1 & -0.060496 & 2.600902 & -0.406812 \\ 1 & 0.171694 & -0.216136 & -1.338072 \\ 7 & 1.539404 & -0.993955 & 0.077031 \\ 9 & 2.455623 & 0.148757 & -0.140117\end{array}$

34. $\underline{\mathrm{TS} 3}$

$\begin{array}{rrrr}6 & 0.739321 & 0.758518 & 0.569194 \\ 6 & 0.739322 & -0.758515 & 0.569196 \\ 7 & -0.437190 & 0.000002 & 0.147894 \\ 1 & 0.609766 & 1.204422 & 1.555110 \\ 1 & 0.609765 & -1.204414 & 1.555114 \\ 6 & 1.466267 & 1.608867 & -0.436117 \\ 6 & 1.466271 & -1.608867 & -0.436113 \\ 1 & 0.958165 & 2.570693 & -0.550086 \\ 1 & 2.491842 & 1.799686 & -0.105404 \\ 1 & 1.503050 & 1.126321 & -1.414702 \\ 1 & 1.503076 & -1.126317 & -1.414694 \\ 1 & 2.491839 & -1.799701 & -0.105388 \\ 1 & 0.958161 & -2.570688 & -0.550095 \\ 1 & -1.265700 & -0.000002 & -0.878422 \\ 7 & -1.673558 & 0.000003 & 0.825595 \\ 9 & -2.394646 & -0.000005 & -0.722535\end{array}$


35. $\underline{\text { Int3 }}$

$\begin{array}{lrcc}6 & 0.777404 & -0.180967 & 0.612135 \\ 6 & -0.777407 & -0.180962 & 0.612136 \\ 7 & 0.000003 & 0.963423 & 0.104852 \\ 1 & 1.222626 & -0.007232 & 1.591768 \\ 1 & -1.222628 & -0.007226 & 1.591769 \\ 6 & 1.609875 & -0.973463 & -0.358039 \\ 6 & -1.609880 & -0.973455 & -0.358040 \\ 1 & 2.582730 & -0.492423 & -0.490603 \\ 1 & 1.774227 & -1.990845 & 0.010472 \\ 1 & 1.130493 & -1.030783 & -1.337315 \\ 1 & -1.130525 & -1.030723 & -1.337332 \\ 1 & -1.774187 & -1.990855 & 0.010440 \\ 1 & -2.582755 & -0.492443 & -0.490560 \\ 7 & 0.000007 & 2.021664 & -0.475964\end{array}$

36. TS4

$\begin{array}{lrrl}6 & -0.258251 & 0.533574 & 0.541378 \\ 6 & 1.075351 & -0.190287 & 0.720948 \\ 7 & -0.910900 & -0.686191 & 0.190174 \\ 1 & -0.669385 & 0.767170 & 1.531821 \\ 1 & 1.638496 & 0.008209 & 1.631649 \\ 6 & -0.417213 & 1.723094 & -0.400952 \\ 6 & 1.826776 & -0.599871 & -0.506915 \\ 1 & -1.449746 & 2.080199 & -0.452026 \\ 1 & 0.219476 & 2.530435 & -0.024210 \\ 1 & -0.079916 & 1.459980 & -1.406341 \\ 1 & 1.155561 & -0.989646 & -1.283423 \\ 1 & 2.401977 & 0.224743 & -0.961153 \\ 1 & 2.540518 & -1.397118 & -0.276634 \\ 7 & -1.820095 & -1.239956 & -0.316807\end{array}$

37. cis-2-butene

$\begin{array}{lrrr}6 & 0.671535 & 0.664987 & 0.000039 \\ 6 & -0.671535 & 0.664987 & -0.000075 \\ 6 & -1.588104 & -0.522937 & 0.000004 \\ 6 & 1.588104 & -0.522936 & -0.000007 \\ 1 & 1.170388 & 1.633728 & 0.000024 \\ 1 & -1.170387 & 1.633730 & 0.000080 \\ 1 & -1.047241 & -1.470687 & -0.004047 \\ 1 & -2.242704 & -0.505506 & -0.878178 \\ 1 & -2.237281 & -0.509841 & 0.882313 \\ 1 & 1.047237 & -1.470692 & 0.001841 \\ 1 & 2.238819 & -0.508646 & -0.881153 \\ 1 & 2.241171 & -0.506694 & 0.879349\end{array}$


38. Nitrogen

$\begin{array}{rrrr}7 & 0.000000 & 0.000000 & 0.565364 \\ 7 & 0.000000 & 0.000000 & -0.565364\end{array}$

39. $\underline{\mathrm{HF}}$
$\begin{array}{llll}9 & 0.000000 & 0.000000 & 0.093390\end{array}$
$\begin{array}{lllll}1 & 0.000000 & 0.000000 & -0.840510\end{array}$

II. B3LYP/6-31G(d) optimized geometries in the high lying path

1. $\underline{\text { TS1a }}$

$\begin{array}{lrrr}6 & 1.079667 & -0.615502 & -0.585542 \\ 6 & 0.864455 & 0.823555 & -0.163124 \\ 7 & 0.221581 & -0.266151 & 0.498281 \\ 1 & 0.548666 & -0.907796 & -1.499363 \\ 1 & 0.214779 & 1.411891 & -0.818477 \\ 6 & 2.344860 & -1.412685 & -0.327601 \\ 6 & 1.882796 & 1.662587 & 0.587851 \\ 1 & 2.104103 & -2.475674 & -0.212286 \\ 1 & 3.034582 & -1.311573 & -1.173639 \\ 1 & 2.856543 & -1.084164 & 0.580584 \\ 1 & 2.535030 & 1.048949 & 1.214523 \\ 1 & 2.504531 & 2.224282 & -0.119040 \\ 1 & 1.372305 & 2.382903 & 1.237183 \\ 1 & -0.711075 & -0.569369 & 0.995716 \\ 7 & -1.780317 & -0.278704 & -0.756081 \\ 1 & -2.622150 & -0.770861 & -0.425398 \\ 9 & -2.030755 & -0.892641 & 1.218599 \\ 9 & -2.186670 & 1.016827 & -0.668010\end{array}$

2. $\underline{\text { TS2a }}$

$\begin{array}{lrrc}6 & -0.860040 & -0.672281 & 0.472568 \\ 6 & -0.252615 & 0.706036 & 0.431495 \\ 7 & 0.243453 & -0.352150 & -0.440440 \\ 6 & -2.208587 & -1.041107 & -0.095578 \\ 6 & -0.902358 & 1.919787 & -0.189401 \\ 7 & 1.663733 & -0.993099 & -0.176970 \\ 9 & 2.447812 & 0.182390 & 0.125230 \\ 1 & -0.508412 & -1.294772 & 1.295168 \\ 1 & 0.451487 & 0.921819 & 1.232123 \\ 1 & 0.880183 & -0.612414 & -1.321820 \\ 1 & -2.215196 & -2.090217 & -0.411536 \\ 1 & -2.983993 & -0.914852 & 0.668536\end{array}$




$\begin{array}{rrrr}1 & -2.475059 & -0.425155 & -0.958513 \\ 1 & -1.564400 & 1.657506 & -1.018927 \\ 1 & -1.489738 & 2.456789 & 0.564070 \\ 1 & -0.133886 & 2.601909 & -0.568807\end{array}$

3. Int2a

$\begin{array}{lrrr}6 & -1.030307 & 0.296592 & -0.867524 \\ 6 & -0.889204 & -0.986174 & -0.128685 \\ 7 & 0.185027 & 0.028531 & -0.039197 \\ 1 & -0.836801 & 0.222098 & -1.935972 \\ 1 & -0.615148 & -1.836582 & -0.749849 \\ 6 & -1.892016 & 1.462005 & -0.448297 \\ 6 & -1.598600 & -1.341374 & 1.154949 \\ 1 & -1.428416 & 2.407191 & -0.751628 \\ 1 & -2.874210 & 1.394977 & -0.929927 \\ 1 & -2.044586 & 1.493960 & 0.633615 \\ 1 & -1.818991 & -0.458861 & 1.761086 \\ 1 & -2.542809 & -1.852724 & 0.935527 \\ 1 & -0.975406 & -2.015122 & 1.752668 \\ 1 & 0.924436 & 1.211938 & 0.931504 \\ 7 & 1.307249 & -0.314054 & -0.884346 \\ 1 & 1.957551 & 0.452744 & -0.669497 \\ 9 & 1.632043 & 1.867133 & 0.975406 \\ 9 & 1.953424 & -1.379050 & -0.172670\end{array}$

4. TS3a

$\begin{array}{lrrr}6 & -0.214833 & 0.748694 & -0.317381 \\ 6 & -0.746399 & -0.691564 & -0.489586 \\ 7 & 0.203604 & -0.339820 & 0.549928 \\ 6 & -1.057798 & 1.884040 & 0.210735 \\ 6 & -2.148434 & -1.115291 & -0.125077 \\ 7 & 1.332913 & -0.818156 & 0.850283 \\ 9 & 2.573152 & 0.064760 & -0.543661 \\ 1 & 0.614529 & 1.011096 & -0.972460 \\ 1 & -0.273926 & -1.257725 & -1.291593 \\ 1 & -0.419274 & 2.628716 & 0.698082 \\ 1 & -1.575526 & 2.378290 & -0.618724 \\ 1 & -1.805767 & 1.551760 & 0.935482 \\ 1 & -2.536478 & -0.563150 & 0.734616 \\ 1 & -2.822037 & -0.954638 & -0.973950 \\ 1 & -2.164155 & -2.182110 & 0.122734 \\ 1 & 2.073433 & -0.044519 & 0.785139\end{array}$

5. $\underline{\mathrm{TS} 3 \mathrm{~b}}$
6
$-0.665596$
$-0.704839$
0.588242
6
$-0.493891$
0.775768
0.495736
0.354836
0.134788
$-0.523159$ 


$\begin{array}{lrrr}6 & -1.489227 & -1.681793 & -0.174040 \\ 6 & -1.470609 & 1.736885 & -0.129583 \\ 7 & 1.392367 & -0.696177 & -0.162680 \\ 9 & 2.481284 & 0.271990 & 0.091204 \\ 1 & -0.145671 & -1.147722 & 1.430446 \\ 1 & 0.085705 & 1.196159 & 1.321197 \\ 1 & 0.304225 & 0.590980 & -1.474009 \\ 1 & -0.802798 & -2.503602 & -0.421998 \\ 1 & -2.287548 & -2.099283 & 0.448920 \\ 1 & -1.892866 & -1.282887 & -1.104872 \\ 1 & -1.778885 & 1.379229 & -1.118304 \\ 1 & -2.362419 & 1.891615 & 0.481061 \\ 1 & -0.965786 & 2.701204 & -0.264539\end{array}$

6. Int3b

$\begin{array}{lrrr}7 & 0.264128 & -0.814455 & 0.877334 \\ 7 & 1.128967 & 0.236349 & 0.525634 \\ 6 & 0.052738 & 0.723623 & -0.432479 \\ 6 & -0.562935 & -0.708337 & -0.346886 \\ 1 & 0.760554 & -1.670379 & 1.119311 \\ 9 & 2.114294 & -0.372852 & -0.472322 \\ 6 & -0.732459 & 1.880473 & 0.160795 \\ 1 & -0.082953 & 2.755812 & 0.264714 \\ 1 & -1.567011 & 2.150846 & -0.496265 \\ 1 & -1.132245 & 1.633643 & 1.148300 \\ 6 & -2.053170 & -0.923419 & -0.163063 \\ 1 & -2.592548 & -0.675722 & -1.085004 \\ 1 & -2.263553 & -1.974130 & 0.069498 \\ 1 & -2.451213 & -0.312108 & 0.652035 \\ 1 & 0.501573 & 0.990810 & -1.388869 \\ 1 & -0.177956 & -1.330395 & -1.163804\end{array}$

7. $\underline{\mathrm{TS} 4 \mathrm{~b}}$

$\begin{array}{lrrc}7 & -0.646380 & 0.487910 & 0.799791 \\ 7 & -0.716797 & -0.794314 & 0.617265 \\ 6 & 0.363091 & -0.771418 & -0.411579 \\ 6 & 0.266822 & 0.785748 & -0.334897 \\ 1 & -1.658423 & 0.821326 & 0.778658 \\ 9 & -2.443798 & -0.020832 & -0.421650 \\ 6 & 1.631416 & -1.506684 & -0.008195 \\ 1 & 1.419547 & -2.573315 & 0.117746 \\ 1 & 2.392026 & -1.399522 & -0.789170 \\ 1 & 2.042984 & -1.127045 & 0.931812 \\ 6 & 1.472252 & 1.653751 & -0.038613 \\ 1 & 2.127354 & 1.693475 & -0.916094 \\ 1 & 1.160051 & 2.678595 & 0.189935 \\ 1 & 2.052037 & 1.277187 & 0.809434 \\ 1 & -0.055799 & -1.181559 & -1.334400\end{array}$


8. $\underline{\text { Int } 4 \mathrm{~b}}$

$\begin{array}{lrcc}7 & -0.847528 & -0.518363 & 0.214444 \\ 7 & -0.729163 & -0.065848 & 1.384698 \\ 6 & 0.756306 & 0.122312 & 1.296697 \\ 6 & 0.594888 & -0.441618 & -0.152239 \\ 1 & -1.834335 & -0.952783 & -1.188641 \\ 9 & -1.872965 & -1.128665 & -2.126756 \\ 6 & 1.222010 & 1.548144 & 1.539153 \\ 1 & 1.040761 & 1.829139 & 2.581786 \\ 1 & 2.296396 & 1.635606 & 1.343019 \\ 1 & 0.695207 & 2.262594 & 0.899696 \\ 6 & 0.869401 & 0.391336 & -1.394040 \\ 1 & 1.937701 & 0.393084 & -1.634535 \\ 1 & 0.311627 & -0.046919 & -2.226855 \\ 1 & 0.537583 & 1.427002 & -1.272781 \\ 1 & 1.257483 & -0.566386 & 1.988049 \\ 1 & 0.995467 & -1.454915 & -0.280359\end{array}$

9. $\underline{\mathrm{TS} 5 \mathrm{~b}}$

$\begin{array}{rrrr}7 & -1.486158 & -0.479446 & 0.459169 \\ 7 & -1.475027 & -1.136342 & -0.518676 \\ 6 & 0.807528 & -0.598165 & -0.056011 \\ 6 & -0.181914 & 0.354064 & 0.523078 \\ 6 & 2.248603 & -0.211110 & -0.081232 \\ 1 & 0.600354 & -1.650080 & 0.062448 \\ 1 & 0.097882 & 0.711715 & 1.536856 \\ 6 & -0.544561 & 1.595302 & -0.300319 \\ 1 & 2.588385 & 0.071593 & 0.935097 \\ 1 & 2.887515 & -1.032762 & -0.419009 \\ 1 & 2.448417 & 0.662247 & -0.718339 \\ 1 & 0.313586 & 2.276868 & -0.320467 \\ 1 & -0.793254 & 1.308024 & -1.325562 \\ 1 & -1.392520 & 2.122363 & 0.152440\end{array}$

10. trans-2-butene

$\begin{array}{lrrc}6 & 0.538086 & 0.395387 & 0.000005 \\ 6 & -0.538086 & -0.395369 & 0.000026 \\ 6 & 1.963499 & -0.079229 & -0.000011 \\ 6 & -1.963500 & 0.079215 & 0.000011 \\ 1 & 0.392428 & 1.477746 & 0.000061 \\ 1 & -0.392434 & -1.477731 & -0.000016 \\ 1 & 2.022729 & -1.173151 & -0.000047 \\ 1 & 2.507892 & 0.289110 & -0.880396 \\ 1 & 2.507903 & 0.289005 & 0.880424\end{array}$




$\begin{array}{rrrr}1 & -2.022750 & 1.173140 & -0.000209 \\ 1 & -2.507816 & -0.289185 & -0.880421 \\ 1 & -2.507948 & -0.288961 & 0.880421\end{array}$

11. Nitrogen
7
$\begin{array}{lll}0.000000 & 0.000000 & 0.552800\end{array}$
7
$\begin{array}{llll}0.000000 & 0.000000 & -0.552800\end{array}$

12. $\underline{\mathrm{HF}}$
9
1
$\begin{array}{rrr}0.000000 & 0.000000 & 0.093380 \\ 0.000000 & 0.000000 & -0.840421\end{array}$

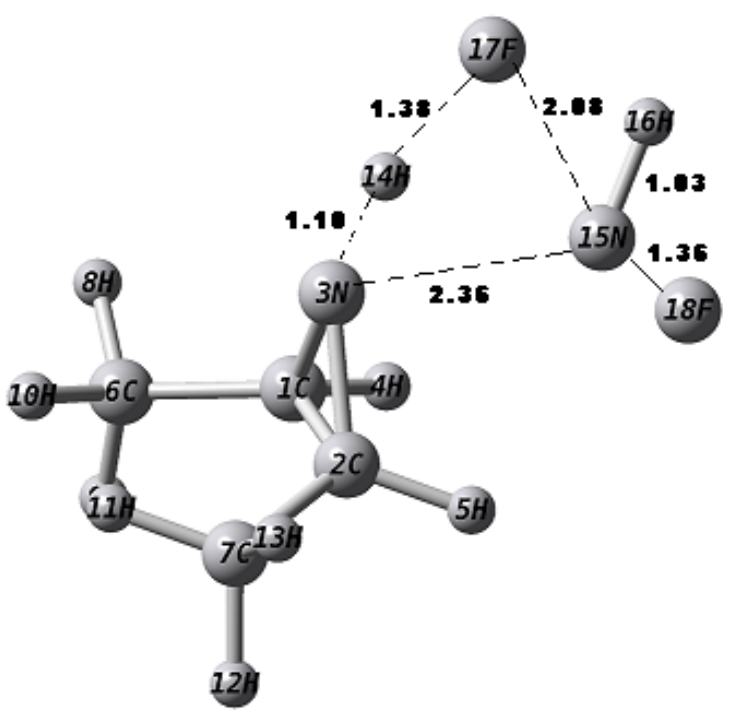

TS1a

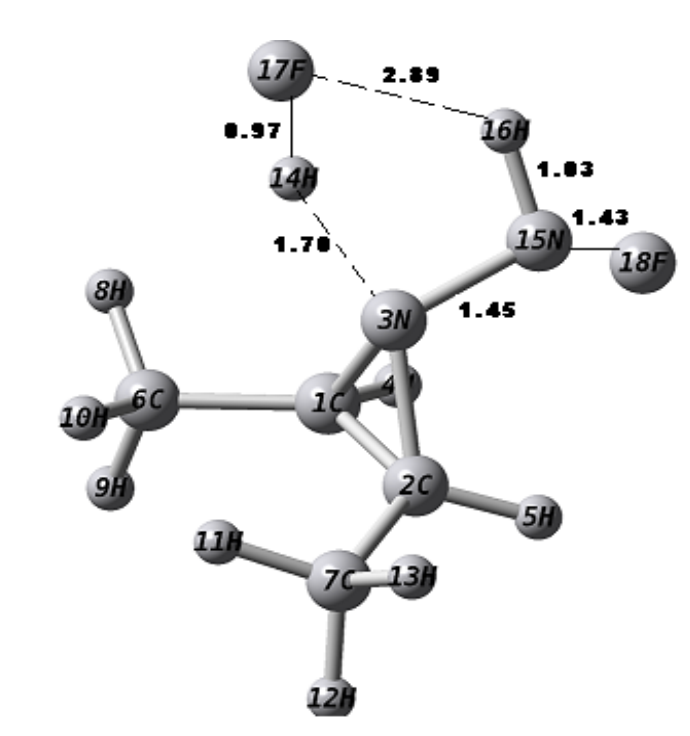

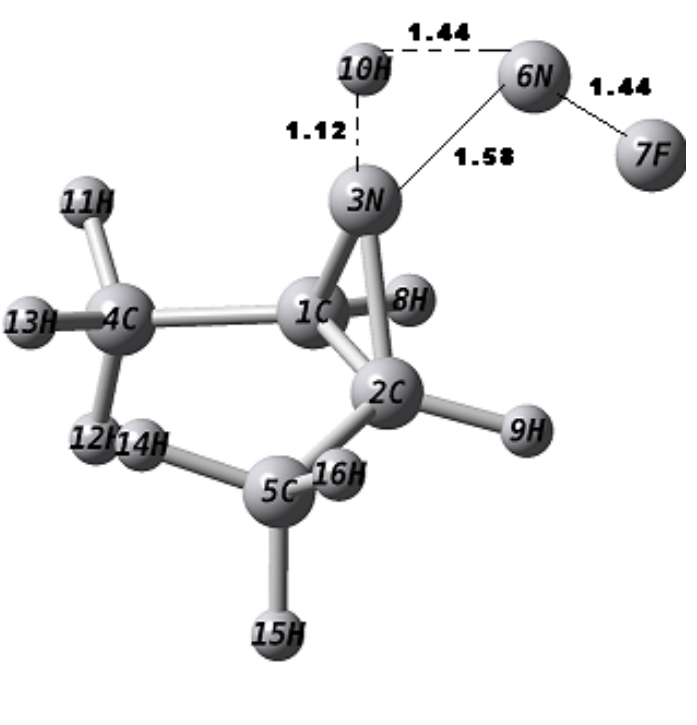

TS2a

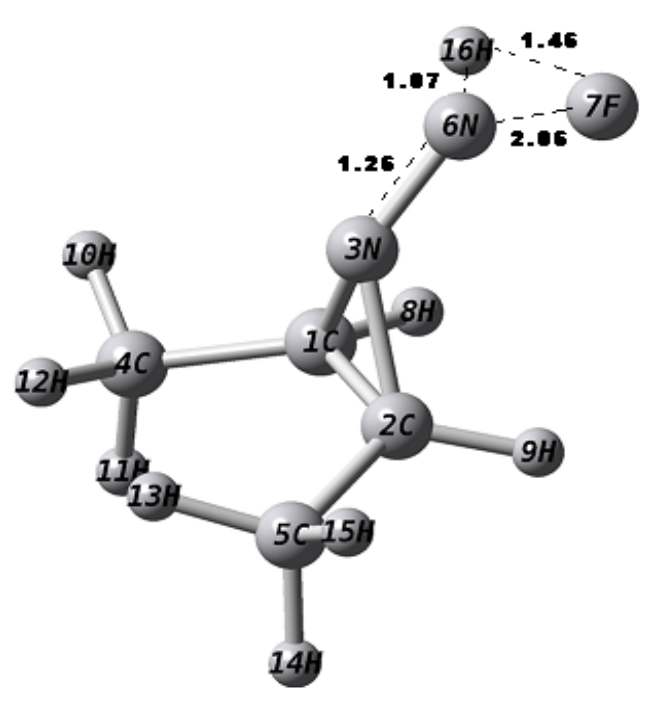



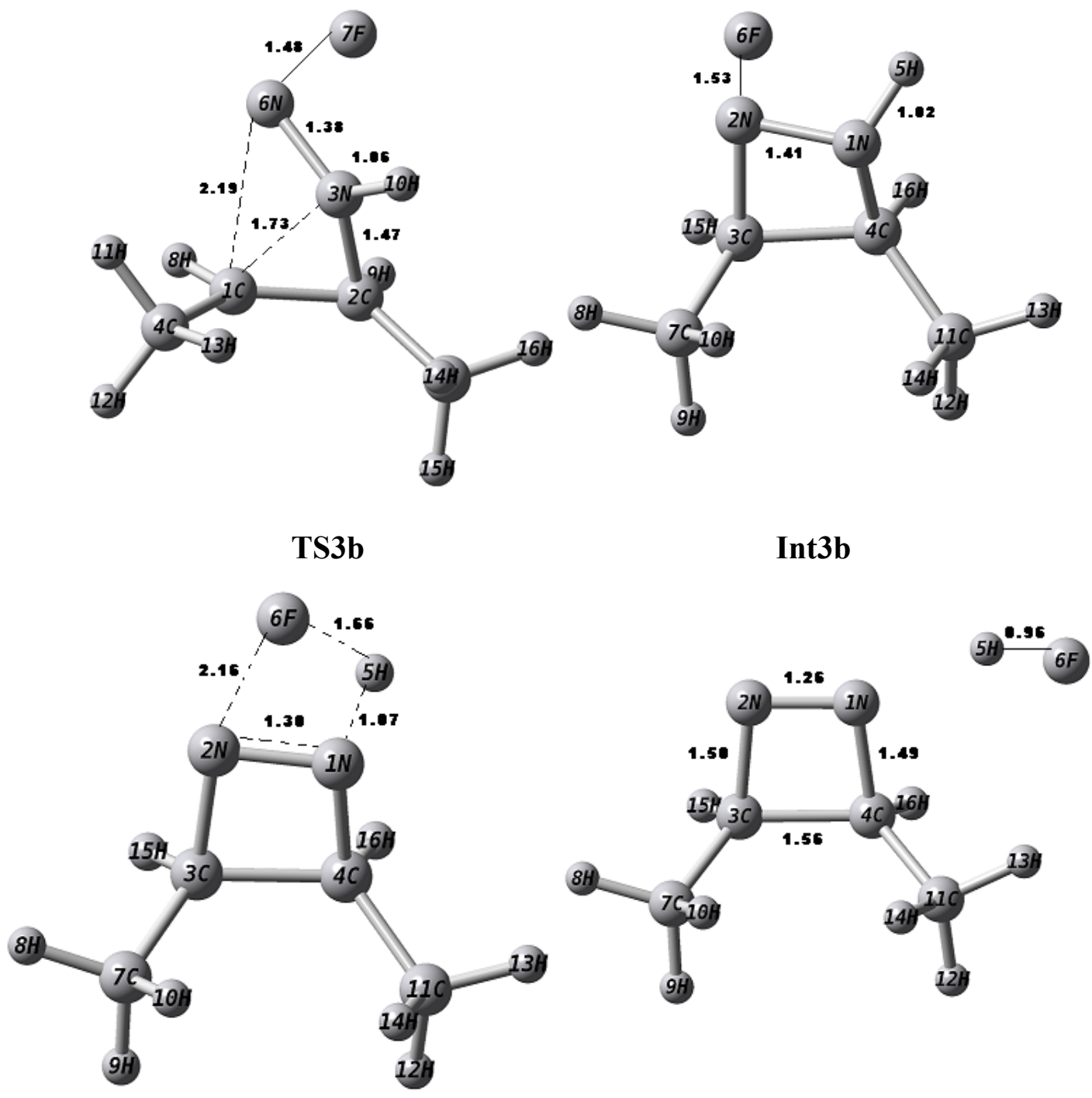

TS4b

Int4b

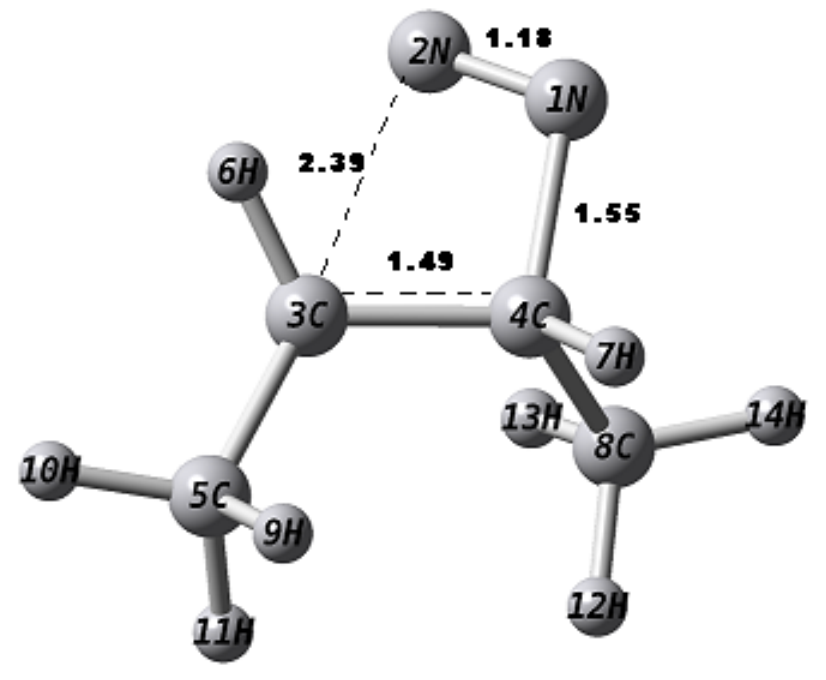

TS5b 